\title{
Prolonged cardiac repolarisation during spontaneous nocturnal hypoglycaemia in children and adolescents with type 1 diabetes
}

\author{
N. P. Murphy ${ }^{1}$ M. E. Ford-Adams ${ }^{1}$ K. K. Ong ${ }^{2}$ N. D. Harris ${ }^{3}$ - S. M. Keane ${ }^{1}$ C. Davies $^{4}$ R. H. Ireland ${ }^{4}$ \\ I. A. MacDonald ${ }^{5}$ E. J. Knight ${ }^{2} \cdot$ J. A. Edge ${ }^{1} \cdot$ S. R. Heller ${ }^{4}$ D. B. Dunger ${ }^{2}$ \\ ${ }^{1}$ Department of Paediatrics, University of Oxford, UK \\ 2 Department of Paediatrics, University of Cambridge, Level 8, Addenbrooke's Hospital, Cambridge, UK \\ ${ }^{3}$ Department of Medical Sciences, University of Bath, UK \\ ${ }^{4}$ Clinical Sciences Centre, University of Sheffield, UK \\ ${ }^{5}$ Department of Physiology, Queen's Medical Centre, University of Nottingham, UK
}

\section{Abstract}

Aims/hypothesis. It has been postulated that hypoglycaemia-related cardiac dysrhythmia and, in particular, prolonged cardiac repolarisation, may contribute to increased mortality rates in children and adolescents with type 1 diabetes.

Methods. We examined the prevalence of prolonged QT interval on ECG during spontaneous hypoglycaemia in 44 type 1 diabetic subjects (aged 7-18 years), and explored the relationships between serial overnight measurements of QT interval corrected for heart rate $(\mathrm{QTc})$ and serum glucose, potassium and epinephrine levels. Each subject underwent two overnight profiles; blood was sampled every $15 \mathrm{~min}$ for glucose measurements and hourly for potassium and epinephrine. Serial ECGs recorded half-hourly between 23.00 and 07.00 hours were available on 74 nights: 29 with spontaneous hypoglycaemia (defined as blood glucose $<3.5 \mathrm{mmol} / \mathrm{l}$ ) and 45 without hypoglycaemia.
Results. Mean overnight QTc was longer in females than in males (412 vs $400 \mathrm{~ms}, p=0.02$ ), but was not related to age, diabetes duration or $\mathrm{HbA}_{1} \mathrm{c}$. Prolonged QTc (>440 ms) occurred on 20 out of $74(27 \%)$ nights, with no significant differences between male and female subjects, and was more prevalent on nights with hypoglycaemia $(13 / 29,44 \%)$ than on nights without $(7 / 45,15 \%, p=0.0008)$. Potassium levels were lower on nights when hypoglycaemia occurred (minimum potassium 3.4 vs $3.7 \mathrm{mmol} / \mathrm{l}, p=0.0003$ ) and were inversely correlated with maximum QTc $(r=-0.40$, $p=0.03$ ). In contrast, epinephrine levels were not higher on nights with hypoglycaemia and were not related to QTc.

Conclusions/interpretation. In young type 1 diabetic subjects, prolonged QTc occurred frequently with spontaneous overnight hypoglycaemia and may be related to insulin-induced hypokalaemia.

Keywords Child $\cdot$ Hypoglycaemia $\cdot$ Hypokalaemia QT interval · Type 1 diabetes
Received: 29 April 2004 / Accepted: 18 July 2004 Published online: 17 November 2004

(C) Springer-Verlag 2004

D. B. Dunger (

Department of Paediatrics,

University of Cambridge,

Level 8, Addenbrooke's Hospital,

Box 116, Cambridge,

CB2 2QQ, UK

E-mail: dbd25@cam.ac.uk

Tel.: +44-1223-336885, Fax: +44-1223-336996

Abbreviations: QTc, corrected QT interval

\section{Introduction}

Children and young people with type 1 diabetes have a higher mortality than their non-diabetic peers [1]. The majority of these deaths are due to diabetic ketoacidosis and associated cerebral oedema, but circumstantial evidence suggests that a proportion are caused by nocturnal hypoglycaemia, the so-called "dead-inbed" phenomenon [2]. The mechanisms linking hypoglycaemia with sudden death are unknown, but could be related to abnormalities in cardiac rhythm.

Observational data supported by experimental studies using hyperinsulinaemic glucose clamps have 
shown that the QT interval is prolonged during periods of hypoglycaemia [3]. In normal subjects, prolongation of the QT interval is associated with an excess mortality risk $[4,5]$, and in patients with coronary artery disease, heart failure and peripheral vascular disease, QT interval prolongation increases the risk of developing ventricular tachyarrhythmias and sudden cardiac death $[6,7,8]$. Abnormal ventricular repolarisation with prolongation of the QT interval during episodes of nocturnal hypoglycaemia therefore offers a mechanism by which hypoglycaemia could induce ventricular tachyarrhythmias and thus contribute to the increased mortality rates in young patients with type 1 diabetes [8].

However, there are as yet no data to link prolonged QT interval with spontaneous hypoglycaemia in young people, the population who are at greatest risk of sudden death [1]. The aims of this prospective study were to determine the effect of age, sex, puberty stage, duration of diabetes and hypoglycaemia on the duration of the corrected QT interval (QTc) in children and adolescents with type 1 diabetes, and to examine the dynamic relationship between QTc and epinephrine and potassium levels during spontaneous hypoglycaemia.

\section{Subjects and methods}

Subjects. We prospectively recruited 44 subjects with type 1 diabetes for longer than 1 year, or who were Cpeptide negative from the Paediatric Diabetes clinics at the John Radcliffe Hospital (Oxford, UK) and Addenbrooke's Hospital (Cambridge, UK) for studies of nocturnal hypoglycaemia $[9,10]$. Each subject was studied on two occasions. ECG data were available for 44 individuals and 74 study nights. The clinical characteristics of the study cohort are displayed in Table 1. Subjects had no complications of diabetes, they were not obese (BMI <98th percentile for age), and none had a family history of prolonged QT syndrome. Patients with chronic disease (other than those with hypothyroidism who were receiving adequate replacement therapy) and patients with unstable metabolic control (defined by $\mathrm{HbA}_{1} \mathrm{c}>12 \%$ ) were excluded. None of the children were on any medications known to cause QT prolongation. The study was approved by the local research ethics committees, and written informed consent was obtained from patients and, where appropriate, from their parents.

Study design. Subjects were admitted for two overnight profiles, performed 16 weeks apart, during which $\mathrm{HbA}_{1} \mathrm{c}$, serial glucose, epinephrine and potassium levels and QTc duration were measured. Prior to admission for the overnight studies, patients were maintained on their usual insulin regimen. All of the adolescents used multiple injection therapy (pre-prandial short-act-
Table 1. Clinical characteristics of the studied children and adolescents with type 1 diabetes

\begin{tabular}{lll}
\hline & $\begin{array}{l}\text { Pre-pubertal } \\
(n=19)\end{array}$ & $\begin{array}{l}\text { Pubertal } \\
(n=25)\end{array}$ \\
\hline Males & 14 & 11 \\
Age (years) & $9.2(7-11)$ & $14.8(12-18)$ \\
Duration of diabetes (years) & $4.1(1-9)$ & $7.3(1.8-15)$ \\
$\mathrm{HbA}_{1} \mathrm{c}(\%)$ & $8.1(7.3-10.7)$ & $9.3(7.1-12)$ \\
Insulin dose $\left(\mathrm{U} \cdot \mathrm{kg}^{-1} \cdot \mathrm{day}^{-1}\right)$ & $0.9(0.5-1.3)$ & $1.2(0.3-1.7)$ \\
\hline
\end{tabular}

Values shown are medians (ranges) or $n$

ing insulin and pre-bed intermediate-acting insulin), and the pre-pubertal children received three injections per day (a mixture of short- and intermediate-acting insulin pre-breakfast, short-acting insulin alone preevening meal, and intermediate-acting insulin alone pre-bed). Of the 74 overnight profiles, 31 were performed while patients were on insulin analogues (18 adolescent nights and 13 pre-pubertal nights).

Overnight metabolic profiles and ECG monitoring. On the day of each scheduled overnight profile, patients were asked to undertake their usual activities, meals and insulin regimen until they were admitted to hospital at 18.00 hours. After application of ethyl chloride local anaesthetic spray, an intravenous cannula was inserted into a hand vein for intermittent venous sampling. Subjects received their short-acting insulin prior to a standard evening meal (50\% carbohydrate, $15 \%$ protein and $35 \%$ fat), and had a snack before receiving their pre-bed insulin at 22.00 hours. Evening meals and snacks were adjusted to their usual intake of carbohydrate, as established by a dietician. Subjects went to bed at 22.30 hours and were encouraged to sleep. $\mathrm{HbA}_{1} \mathrm{c}$ levels were measured on admission. Venous blood samples were taken every $15 \mathrm{~min}$ for measurement of blood glucose levels (assayed the next day), and samples for assessment of free insulin, epinephrine and potassium levels were taken hourly.

When preparing for bed, subjects were attached to a custom-built three-lead ECG monitoring system. This consisted of a laptop computer and a low-noise, high-gain $(\times 10,000)$ three-channel ECG amplifier (bandwidth $0.5-125 \mathrm{~Hz}$ ) with $9 \mathrm{~V}$ battery supply, 12bit $\mathrm{AD}$ converter at a sampling rate of $250 \mathrm{~Hz}$ and an isolated RS 232 digital interface. A 20-s ECG recording was made every $30 \mathrm{~min}$ during sleep from 23.00 to 07.00 hours. At the end of each night the data were analysed using a custom-built software package. Each 20 -s recording was signal-averaged using a template matching method. QT interval was measured from the beginning of the $\mathrm{Q}$ wave to the end of the $\mathrm{T}$ wave using the intersection of the $\mathrm{T}$ wave with the isoelectric line [11]. The QT intervals were corrected for heart rate using Bazett's formula to generate QTc values $[12,13]$. 
Assays. Blood glucose levels were measured the day after the overnight profile in $25-\mu l$ samples of whole blood that had been collected into bottles containing heparin/fluoride using an YSI model 2300 Stat Plus Analyser (YSI, Lynchford House, Farnborough, UK). At a concentration of $4.4 \mathrm{mmol} / \mathrm{l}$, the intra-assay $\mathrm{CV}$ was $2.6 \%$ and the inter-assay CV was $3.1 \%$.

Blood samples for catecholamine analysis were added to EGTA/glutathione preservative, spun for $10 \mathrm{~min}$ and then separated immediately before being stored at $-70{ }^{\circ} \mathrm{C}$. Epinephrine was extracted from 50$\mu \mathrm{l}$ samples of plasma using an organic solvent method and separated and quantified by HPLC with electrochemical detection [14]. The intra-assay CV was $9.9 \%$ and the inter-assay $\mathrm{CV}$ was $8.5 \%$.

Serum potassium levels were measured by indirect potentiometry. The intra-assay and inter-assay $\mathrm{CVs}$ were $2 \%$ and $4 \%$ respectively.

$\mathrm{HbA}_{1} \mathrm{c}$ levels were determined by HPLC (Clinserv Laboratories, Hamburg, Germany). The intra-assay CVs were $1.53 \%$ and $0.94 \%$ at $\mathrm{HbA}_{1} \mathrm{c}$ levels of $5.9 \%$ and $14.4 \%$ respectively. The inter-assay CVs were $1.3 \%$ and $1.8 \%$ at $\mathrm{HbA}_{1} \mathrm{c}$ levels of $5.4 \%$ and $9.7 \%$ respectively.

Free insulin levels were determined by RIA only in patients on conventional insulins $(n=37)$ because of concerns about the sensitivity of the assay for detection of insulin analogues (Glargine and Lispro). The inter-assay CVs were $4.4 \%$ and $2.2 \%$ at concentrations of $1.2 \mathrm{mU} / \mathrm{l}$ and $4.7 \mathrm{mU} / \mathrm{l}$ respectively.

Calculations and statistics. "Prolonged QTc" was defined as a 20-s signal-averaged recording of longer than $440 \mathrm{~ms}$ [15]. Biochemical hypoglycaemia was defined as two consecutive blood glucose levels lower than $3.5 \mathrm{mmol} / \mathrm{l}$. Positively skewed data, such as blood glucose and insulin concentrations, were log transformed to normal distributions to allow use of parametric tests.

Comparisons of QTc and hormone levels between nights with and without hypoglycaemia were performed using unpaired $t$ tests on mean or maximum overnight values. Significant differences between nights with and without hypoglycaemia were confirmed by performing paired $t$ tests in 13 subjects who had one night with hypoglycaemia one night without. Correlation coefficients were determined by linear regression. Data are presented as means \pm SD or medians (ranges), except for demographic data which are presented as means (ranges). Statistics were performed using SPSS Version 10.0 (SPSS, Chicago, Ill., USA).

Cross-correlation was used to determine the interrelationships between overnight changes in QTc and blood glucose and potassium levels. This is an iterative technique that recognises statistically coincident waveforms within a data array [16]. One data array is serially correlated against another with progressive step changes (time lags) in the time relationship between the data arrays. The result is dependent both on the relative amplitude of such waves and pulses (i.e. whether large pulses of one array are associated with large pulses of the other) and on the phase relationship, or time lag, between data arrays. Correlograms derived from individual overnight profiles were pooled using Fisher's $z$ transformation to obtain an overall estimate of the likelihood of the significance and phase relationship of the interrelationship.

\section{Results}

Overnight QTc values. QTc measurements from 74 overnight profiles were available for analysis (39 pubertal, 35 pre-pubertal). Overnight QTc values were longer for female subjects than for male subjects $(412 \pm 9$ vs $400 \pm 24 \mathrm{~ms}, p=0.02)$. However, no differences in maximum QTc were observed with regard to sex. Age, pubertal stage and duration of diabetes were not related to mean or maximum QTc. A "prolonged QTc" ( $>440 \mathrm{~ms}$ ) was noted on 20 of the $74(27.0 \%)$ study nights and was not related to age, sex, $\mathrm{HbA}_{1} \mathrm{c}$ or duration of diabetes.

Overnight glucose levels. Between 23.00 and 07.00 hours, blood glucose levels were $4.3 \pm 1.3 \mathrm{mmol} / \mathrm{l}$ on the nights with hypoglycaemia $(n=29)$ compared with $10.3 \pm 2.6 \mathrm{mmol} / \mathrm{l}$ on the nights without hypoglycaemia $(n=45, p<0.0005)$ (Fig. 1a). The median time of onset of hypoglycaemia was 01:45 hours (range 23:15-06:30) and the median duration of hypoglycaemia was $120 \mathrm{~min}$ (range 15-540). On the nights with hypoglycaemia the median glucose nadir was $2.0 \mathrm{mmol} / \mathrm{l}$ (range 1.1-3.1) and the median time at glucose nadir was 02:30 hours (range 23:45-07:00).

Hypoglycaemia versus non-hypoglycaemia. Insulin doses were similar on nights with and without hypoglycaemia ( $p=0.6$, data not shown). However, mean overnight serum free insulin levels were more than $50 \%$ higher on nights when hypoglycaemia occurred $(n=18)$ than on nights when it did not occur $(n=19$, $p=0.006$ ) (Table 2, Fig. 1b).

Potassium levels were lower on nights with hypoglycaemia than on nights without hypoglycaemia (Table 2, Fig. 1c). Peak epinephrine levels were higher in pubertal adolescents than in prepubertal children $(1.54 \pm 1.31$ vs $0.57 \pm 0.58 \mathrm{nmol} / 1, p=0.002)$; however, overnight hypoglycaemia did not affect peak epinephrine levels in either group (Table 2).

Relationship between QTc and hypoglycaemia. Overnight QTc values were longer on nights when spontaneous hypoglycaemia occurred than on nights when it did not occur (Table 2, Fig. 1d), and "prolonged QTc" (>440 ms) occurred more frequently on 
a

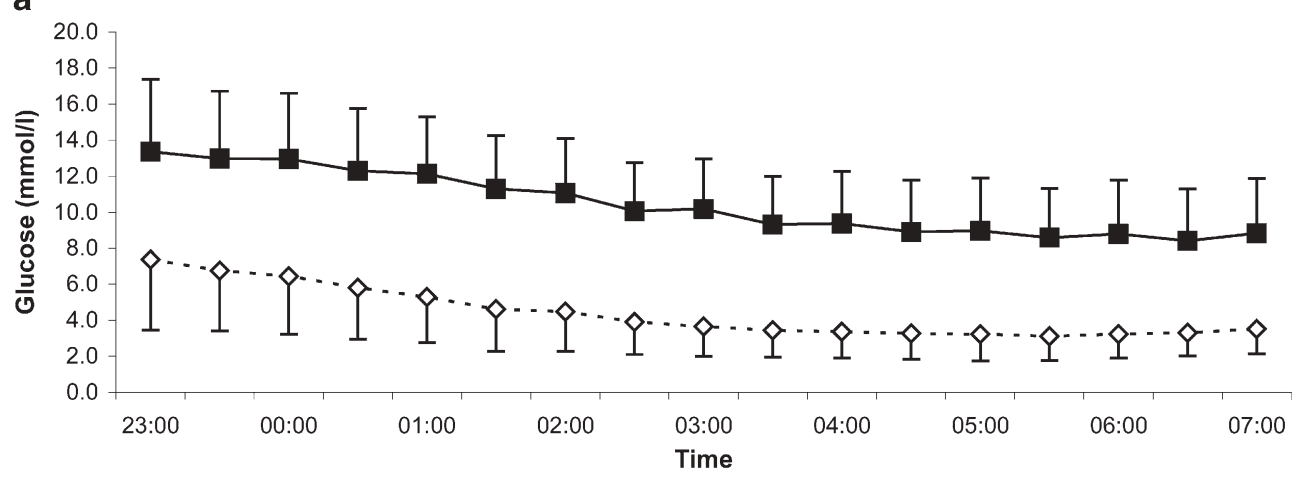

b

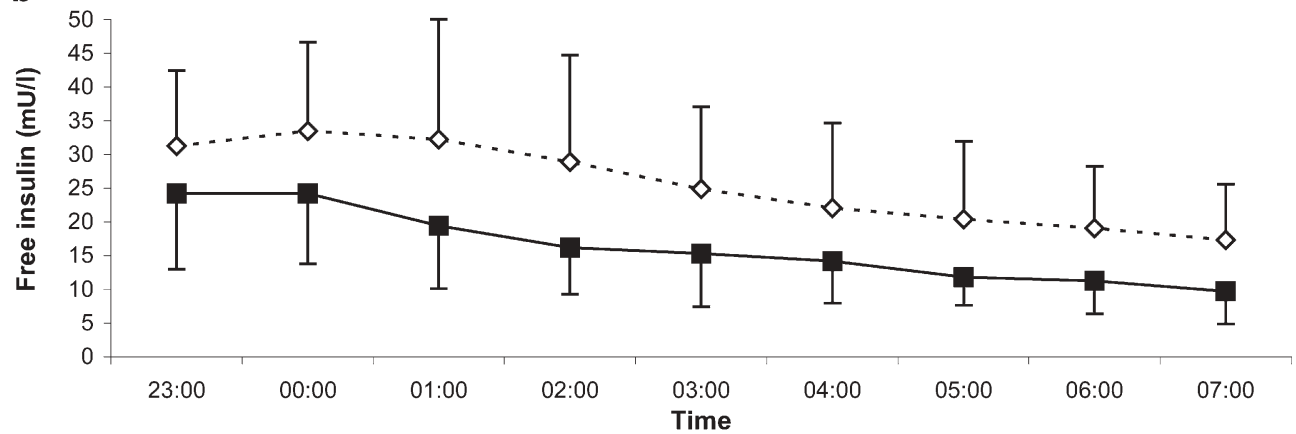

C
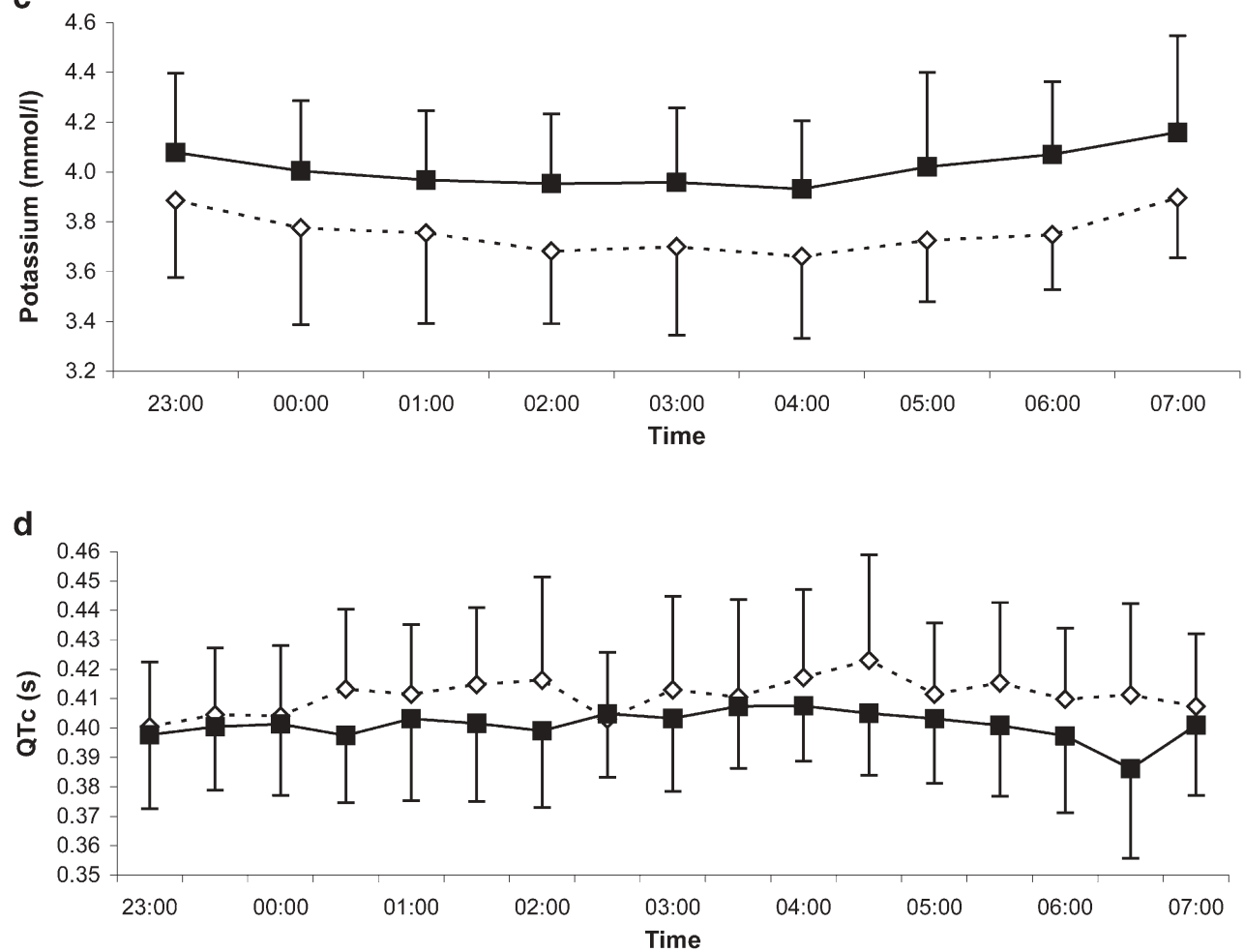

Fig. 1. Overnight measurements of blood glucose (a), free insulin (b), potassium levels (c) and QTc (d) on nights with ( $n=29$; open diamonds and dotted line) and without ( $n=45$; solid squares and solid line) spontaneous hypoglycaemia. Data shown are means \pm SD. Free insulin levels were only measured on nights when subjects were taking non-analogue insulins ( $n=18$ on nights with hypoglycaemia, $n=19$ on nights without hypoglycaemia) nights with spontaneous hypoglycaemia $(13 / 29,44 \%)$ than on those without hypoglycaemia $(7 / 45,15 \%$; $p=0.0008$ ).

As each subject underwent two overnight profiles, we allowed for potential confounding effects of repeated observations in the same individual by taking average QTc values in those subjects who had repeated hypoglycaemia or repeated non-hypoglycaemia. 
Table 2. Overnight QTc, potassium, epinephrine, and free insulin levels on nights with and without hypoglycaemia

\begin{tabular}{|c|c|c|c|c|}
\hline & & $\begin{array}{l}\text { Hypoglycaemia } \\
(n=29)\end{array}$ & $\begin{array}{l}\text { No hypoglycaemia } \\
(n=45)\end{array}$ & $p$ value ( $t$ test) \\
\hline QTc (ms) & $\begin{array}{l}\text { Max. } \\
\text { Mean } \\
\text { Min. }\end{array}$ & $\begin{array}{l}442 \pm 34 \\
412 \pm 22 \\
384 \pm 23\end{array}$ & $\begin{array}{l}424 \pm 18 \\
401 \pm 19 \\
371 \pm 30\end{array}$ & $\begin{array}{l}0.003^{\mathrm{a}} \\
0.03^{\mathrm{a}} \\
0.05^{\mathrm{a}}\end{array}$ \\
\hline Potassium (mmol/l) & $\begin{array}{l}\text { Max. } \\
\text { Mean } \\
\text { Min. }\end{array}$ & $\begin{array}{l}4.1 \pm 0.3 \\
3.8 \pm 0.2 \\
3.4 \pm 0.3\end{array}$ & $\begin{array}{l}4.4 \pm 0.3 \\
4.0 \pm 0.3 \\
3.7 \pm 0.3\end{array}$ & $\begin{array}{l}0.0005^{\mathrm{a}} \\
0.000007^{\mathrm{a}} \\
0.0003^{\mathrm{a}}\end{array}$ \\
\hline Epinephrine (nmol/l) & $\begin{array}{l}\text { Max. } \\
\text { Mean } \\
\text { Min. }\end{array}$ & $\begin{array}{l}1.22 \pm 0.98 \\
0.53 \pm 0.43 \\
0.20 \pm 0.21\end{array}$ & $\begin{array}{l}1.21 \pm 1.17 \\
0.54 \pm 0.64 \\
0.18 \pm 0.23\end{array}$ & $\begin{array}{l}\text { NS } \\
\text { NS } \\
\text { NS }\end{array}$ \\
\hline Free insulin (mU/l) & $\begin{array}{l}\text { Max. } \\
\text { Mean } \\
\text { Min. }\end{array}$ & $\begin{array}{l}37.9 \pm 17.8 \\
24.8 \pm 8.3 \\
16.2 \pm 8.3\end{array}$ & $\begin{array}{c}26.9 \pm 11.2 \\
16.2 \pm 4.2 \\
8.6 \pm 4.2\end{array}$ & $\begin{array}{l}0.03^{\mathrm{a}} \\
0.006^{\mathrm{a}} \\
0.004^{\mathrm{a}}\end{array}$ \\
\hline
\end{tabular}

Values shown are means \pm SD. Hypoglycaemia was defined as blood glucose $<3.5 \mathrm{mmol} / \mathrm{l}$ for at least $15 \mathrm{~min}$. Free insulin levels were measured only on nights when subjects were taking non-analogue insulins ( $n=18$ on nights with hypoglycemia, $n=19$ on nights without hypoglycaemia). Data were also analy- sed making adjustments for repeated measurements in individual subjects who had two nights with $(n=5)$ or without $(n=10)$ hypoglycaemia. a $p<0.05$ when analysed using the average of both overnight profiles in cases with repeated measurements

Table 3. Overnight glucose, potassium, epinephrine and insulin levels on nights with and without prolonged QTc ( $>440 \mathrm{ms)}$

\begin{tabular}{lllll}
\hline & & $\begin{array}{l}\text { Prolonged QTc } \\
(n=20)\end{array}$ & $\begin{array}{l}\text { Normal QTc } \\
(n=54)\end{array}$ & $p$ value $(t$ test $)$ \\
\hline Glucose (mmol/l) & Max. & $10.6 \pm 4.7$ & $12.5 \pm 4.6$ & 0.055 \\
& Mean & $6.9 \pm 3.1$ & $9.2 \pm 3.3$ & 0.005 \\
& Min. & $2.9 \pm 0.5$ & $6.6 \pm 2.6$ & 0.0003 \\
Potassium (mmol/l) & Max. & $4.32 \pm 0.35$ & $4.46 \pm 0.34$ & 0.2 \\
& Mean & $3.85 \pm 0.22$ & $3.97 \pm 0.21$ & 0.04 \\
Epinephrine (nmol/l) & Min. & $3.37 \pm 0.29$ & $3.62 \pm 0.28$ & 0.001 \\
& Max. & $1.41 \pm 1.27$ & $1.07 \pm 1.17$ & 0.3 \\
& Mean & $0.56 \pm 0.56$ & $0.48 \pm 0.52$ & 0.6 \\
& Min. & $0.21 \pm 0.26$ & $0.19 \pm 0.20$ & 0.7 \\
& Max. & $51.5 \pm 21.4$ & $42.2 \pm 4.3$ & 0.2 \\
& Mean & $23.8 \pm 7.8$ & $20.5 \pm 12.0$ & 0.3 \\
& Min. & $10.8 \pm 3.8$ & $8.4 \pm 2.7$ & 0.1
\end{tabular}

Values shown are means \pm SD. a Free insulin levels were only measured on nights when subjects were using non-analogue insulins ( $n=9$ on prolonged QTc nights, $n=26$ on normal QTc nights)

The relationship between hypoglycaemia and prolonged QTc remained significant ("mean QTc" 411 \pm 2 vs $401 \pm 2 \mathrm{~ms}, p=0.04$; "maximum QTc" $442 \pm 3$ vs $424 \pm 2 \mathrm{~ms}, p=0.008$ ). Furthermore, paired analysis of the profiles from 13 individuals who had one night with hypoglycaemia and one night without showed that the mean $(416 \pm 2$ vs $407 \pm 2 \mathrm{~ms}, p=0.002)$ and maximum ( $450 \pm 4$ vs $428 \pm 17 \mathrm{~ms}, p=0.02)$ overnight QTc values were greater on nights with hypoglycaemia.

QTc related to insulin, potassium and epinephrine levels. An inverse relationship was seen between maximum QTc and minimum potassium levels ( $r=$ $-0.40, p=0.03$ ) on nights with spontaneous hypoglycaemia. Thus, potassium levels were lower on nights with prolonged QTe than on nights without prolonged QTc (Table 3). However, no relationship was observed between QTc and overnight insulin levels (Table 3). Similarly, maximum QTc values were not related to maximum epinephrine levels on nights with hypoglycaemia $(r=0.07, p=0.6)$, and epinephrine levels did not differ significantly between nights with and those without prolonged QTc (Table 3 ).

Cross-correlation analysis indicated that overnight changes in QTc coincided with changes in glucose levels, without any apparent time lag $(r=-0.13,95 \%$ CI -0.28 to $0.00, p<0.06)$. Changes in circulating potassium levels also coincided with changes in glucose levels with no time lag $(r=0.25,95 \%$ CI $0.08-0.43$, $p<0.05)$. 


\section{Discussion}

In this study we have shown that children and adolescents with type 1 diabetes frequently exhibit abnormal prolongation of cardiac repolarisation, manifest by QTc on ECG monitoring, particularly on nights when spontaneous hypoglycaemia occurs. The QT interval is an indirect measure of cardiac repolarisation in cardiac myocytes, which shortens as heart rate increases above the physiological range, particularly during childhood [17]. Although there are a number of methods available to correct the QT interval for heart rate (i.e. calculate QTc), Bazett's correction (QT divided by the square root of the RR interval) remains the standard [12, 13], and during sinus rhythm a QTc of $>440 \mathrm{~ms}$ is considered prolonged [15]. In normal subjects, QTc varies with age and is longer in females than males after the onset of puberty [17], possibly due to gradual QT shortening in males [18]. Others have reported that sex differences in QTc are absent during childhood $[19,20]$. In our study we observed a similar difference in QTc with regard to sex among prepubertal and pubertal subjects with type 1 diabetes; however, QTc was not associated with age.

In our study, prolonged QTc commonly occurred in children and adolescents with type 1 diabetes (27\% of all nights). Although we did not include non-diabetic subjects as controls, our findings are consistent with studies in type 1 diabetic adults showing an increased likelihood of prolonged QTc compared with those without diabetes $[21,22,23]$. A recent study of children and adolescents reported a similar increased rate of prolonged QTc during daytime ECG monitoring in type 1 diabetic patients $(23 \%)$ compared with matched control subjects (3\%) [24]. While it may be difficult to compare QTc values between studies [11], figures from a large normative database indicate that prolonged QTc (>440 ms) is unusual in healthy children $[25,26]$. In our subjects, QTc was measured during sleep. A previous study reported that QTc was only $19 \mathrm{~ms}$ longer in sleeping patients than in awake patients with the same heart rate [15]. Based on this, we conclude that children and adolescents with type $1 \mathrm{di}$ abetes have longer mean QTc values and a higher incidence of prolonged QTc than normal subjects.

A novel finding in our study was that prolonged QTc in children and adolescents with type 1 diabetes was closely linked to spontaneous nocturnal hypoglycaemia and to lower circulating potassium levels. Cross-correlation analyses indicated that changes in QTc coincided with changes in glucose and potassium levels. Reductions in both blood glucose and potassium levels are mediated by insulin, and we observed that free insulin levels were approximately $50 \%$ higher with hypoglycaemia than without. This result is consistent with previous observations in young children and adolescents [27]. Although we did not observe a direct relationship between changes in free insulin levels and QTc, this might reflect the time lag between insulin level and maximal insulin activity [16]. The duration of cardiac repolarisation is determined by the balance between inward depolarising and outward repolarising currents during the plateau of the action potential. Potassium transfer plays a major role in this process, and hypokalaemia is associated with prolonged QTc [28]. The increased incidence of prolonged QTc in patients with anorexia nervosa is also associated with a modest decrease in serum potassium levels [29]. Furthermore, prolonged QTc due to quinidine treatment or congestive heart failure may be significantly reduced by a modest elevation in serum potassium with potassium infusions [30]. Thus, the induction of extracellular to intracellular potassium transfer by increased insulin activity could delay the outward repolarising current and thereby explain the association between prolonged QTc and spontaneous hypoglycaemia in type 1 diabetic subjects.

Prolonged QTc in type 1 diabetes has previously been attributed to hyperglycaemia [31], possibly mediated by increased sympathetic activity or increased cytosolic calcium content in cardiac myocytes [31]. High glucose levels may increase the production of free-radicals and reduce target cell nitric oxide availability, inducing a state of increased vasomotor tone and ventricular instability [31]. Reduction of sodium / potassium channel ATPase activity, inhibition of calcium channel ATPase activity, depressed sodium / calcium exchanger activity, and activation of sodium/hydrogen antiport have all been implicated [31]. However, in our study we did not observe an association between $\mathrm{HbA}_{1} \mathrm{c}$ and prolonged QTc.

Alternatively, prolonged QTc could be a manifestation of autonomic dysfunction in type 1 diabetes, which is associated with chronic high glucose levels $[31,32]$. Our young subjects with short duration of diabetes did not display any clinical evidence of autonomic neuropathy or other complications. Conversely, other studies have reported that abnormal autonomic function at this age $[33,34,35,36,37]$ is largely related to hyperglycaemia and elevated $\mathrm{HbA}_{1} \mathrm{c}$. However, our data strongly indicate that spontaneous hypoglycaemia rather than hyperglycaemia is the more important determinant of prolonged QTc at night in type 1 diabetic children and adolescents. We have recently demonstrated similar changes during hypoglycaemia in adults [38]. We chose a glucose level of $3.5 \mathrm{mmol} / \mathrm{l}$ to define hypoglycaemia as previous studies carried out by our group in children and adolescents demonstrated that levels in normal children almost never go below this value. The minimum glucose level was lower than $3.0 \mathrm{mmol} / \mathrm{l}$ on $97 \%$ of the nights with hypoglycaemia $(90 \%$ were $<2.5 \mathrm{mmol} / \mathrm{l})$. Using a cut-off value of $2.5 \mathrm{mmol} / \mathrm{l}$ for hypoglycaemia, prolonged QTc was seen on 13 of the $21(62 \%)$ nights hypoglycaemia occurred and on only seven of the $53(13 \%)$ 
nights it did not occur $(p<0.0001)$. All 13 cases of prolonged QTc with hypoglycaemia had glucose levels lower than $2.5 \mathrm{mmol} / \mathrm{l}$. Indeed, Table 3 shows that our finding is not dependent on the cut-off value used to define hypoglycaemia.

It has been suggested that the lengthening of QTc during hypoglycaemia could be related to the magnitude of the counter-regulatory response [3]. Epinephrine infusion prolongs the QTc in normal subjects independently of changes in potassium levels [39]. Experimental hypoglycaemia induced by insulin infusion prolongs cardiac repolarisation in both non-diabetic and diabetic subjects [40, 41, 42], and this appears to be mediated by sympathoadrenal stimulation acting directly on the myocardium and by decreasing circulating potassium levels [43]. However, the design of these experiments may produce unusually high sympathoadrenal activation. In our study of spontaneous nocturnal hypoglycaemia, counter-regulatory responses were blunted in both prepubertal children and adolescents. Epinephrine levels were not significantly higher on nights with hypoglycaemia, and these findings are in agreement with our previous observations in prepubertal children [44]. Recent data on subjects with and without type 1 diabetes suggest that the nocturnal counter-regulatory response may be reduced during sleep [45].

The clinical significance of our observed changes in ventricular repolarisation is unknown, but could possibly link nocturnal hypoglycaemia to reports of sudden death [40]. Tatersall and Gill first raised the possibility of hypoglycaemia-induced arrhythmias in their original paper on the "dead-in-bed" syndrome [2]. This phenomenon, which refers to sudden death in type 1 diabetes, is rare before 12 years, but the risk increases in the adolescent population and persists until the fourth decade of life [40]. A Norwegian study reported that two-thirds of all diabetes-related sudden deaths occurred in the group aged 12-19 years, with hypoglycaemia implicated as a cause [46], whereas in comparable UK studies, hypoglycaemia was only associated with $8 \%$ of sudden deaths [1].

In our study, prolonged QTc was associated with hypoglycaemia and changes in serum potassium levels; however, some episodes of prolonged QTc occurred on nights without hypoglycaemia. Insulin excess could exacerbate these events, but QTc was not associated with insulin levels or dose in our limited series. Given the high incidences of hypoglycaemia and prolonged QTc observed in this study, and the fortunately rare occurrence of sudden death (2.6 episodes per 10,000 patient-years [46]), other factors must contribute. Epinephrine levels were not increased in our children and, as mentioned above, recent data report that the counter-regulatory response may be blunted during sleep [45]. However, increased insulin levels and decreased potassium levels could still contribute to QTc elevation, and further evaluation of the factors that regulate QTc in type 1 diabetes is needed to determine the significance of these findings.

Acknowledgements. We thank all the patients who participated in these studies and their families. We also thank S. Strang and $\mathrm{K}$. Ross for their valuable advice and support in recruitment, and D. Harris and Z. Madgewick for laboratory support. These studies were supported by research grants from Eli Lilly (Indianapolis, Ind., USA) and Aventis (Strasbourg, France). D.B. Dunger is also supported by the Juvenile Diabetes Research Foundation and the Wellcome Trust. This paper was reported in brief as an oral presentation at the American Diabetes Association Annual Scientific Meeting held in San Francisco (Calif., USA) in June 2002.

\section{References}

1. Edge JE, Ford-Adams M, Dunger DB (1999) Causes of death in children with diabetes 1990-1996. Arch Dis Child $81: 313-324$

2. Tatersall RB, Gill GV (1991) Unexplained deaths of Type 1 diabetic patients. Diabet Med 8:49-58

3. Marques JL, George E, Peacey SR et al. (1997) Altered ventricular repolarization during hypoglycaemia in patients with diabetes. Diabet Med 14:648-654

4. Schouten EG, Dekker JM, Meppelink P, Kok FJ, Vandenbroucke JP, Pool J (1991) QT interval prolongation predicts cardiovascular mortality in an apparently healthy population. Circulation 84:1516-1523

5. Elming H, Holm E, Jun L et al. (1998) The prognostic value of the QT interval and QT interval dispersion in allcause and cardiac mortality and morbidity in a population of Danish citizens. Eur Heart J 19:1391-1400

6. Glancy JM, Garratt CJ, Woods KL, de Bono DP (1995) QT dispersion and mortality after myocardial infarction. Lancet 345:945-948

7. Barr CS, Naas A, Freeman M, Lang CC, Struthers AD (1994) QT dispersion and sudden unexpected death in chronic heart failure. Lancet 343:327-329

8. Darbar D, Luck J, Davidson N et al. (1996) Sensitivity and specificity of QTc dispersion for identification of risk of cardiac death in patients with peripheral vascular disease. BMJ 312:874-879

9. Murphy NP, Keane SM, Ong KK et al. (2003) Randomized cross-over trial of insulin glargine plus lispro or NPH insulin plus regular human insulin in adolescents with type 1 diabetes on intensive insulin regimens. Diabetes Care 3: 799-804

10. Ford-Adams ME, Murphy NP, Moore EJ et al. (2003) Insulin lispro: a potential role in preventing nocturnal hypoglycaemia in young children with diabetes mellitus. Diabet Med 20:656-660

11. Ireland RH, Robinson RT, Heller SR, Marques JL, Harris ND (2000) Measurement of high resolution ECG QT interval during controlled euglycaemia and hypoglycaemia. Physiol Meas 21:295-303

12. Bazett HC (1920) An analysis of the time-relations of electrocardiograms. Heart 7:353-370

13. Schwartz PJ, Moss AJ, Vincent GM, Crampton RS (1993) Diagnostic criteria for the long QT syndrome: an update. Circulation 88:782-784

14. Forster CD, Macdonald IA (1999) The assay of the catecholamine content of small volumes of human plasma. Biomed Chromatogr 13:209-215 
15. Garson A Jr (1993) How to measure the QT interval—what is normal? Am J Cardiol 72:14B-16B

16. Matthews DR, Lang DA, Burnett MA, Turner RC (1983) Control of pulsatile insulin secretion in man. Diabetologia 24:231-237

17. Pearl W (1996) Effects of gender, age, and heart rate on QT intervals in children. Pediatr Cardiol 17:135-136

18. Lehmann MH, Hardy S, Archibald D, Quart B, MacNeil DJ (1996) Sex difference in risk of torsade de points with d,1-sotalol. Circulation 94:2535-2541

19. Lehmann MH, Timothy KW, Frankovich D et al. (1997) Age-gender influence on the rate corrected QT interval and the QT heart rate relation in families with genotypically characterised long QT syndrome. J Am Coll Cardiol 29:93-99

20. Davignon A, Rautaharju P, Boiselle E et al. (1980) Normal ECG standards for infants and children. Pediatr Cardiol $1: 123$

21. Veglio M, Sivieri R, Chinaglia A, Scaglione L, CavalloPerin P (2000) QT Interval prolongation and mortality in Type 1 diabetic patients. Diabetes Care 23:1381-1383

22. Arildsen H, May O, Christiansen EH, Damsgaard EM (1999) Increased QT dispersion in patients with insulin-dependent diabetes mellitus. Int J Cardiol 71:235-242

23. Brown DW, Giles WH, Greenlund KJ, Valdez R, Croft JB (2001) Impaired fasting glucose, diabetes mellitus, and cardiovascular disease risk factors are associated with prolonged QTc duration. Results from the Third National Health and Nutrition Examination Survey. J Cardiovasc Risk 8:227-233

24. Suys BE, Huybrechts SJ, Wolf D et al. (2002) QTc interval prolongation and QTc dispersion in children and adolescents with type 1 diabetes. J Pediatr 141:59-63

25. Eberle T, Hessling G, Ulmer H, Brockmeier K (1998) Prediction of normal QT intervals in children. J Electrocardiol 31:121-125

26. Tutar EH, Ocal B, Imamoglu A, Atalay S (1998) Dispersion of QT and QTc interval in healthy children, and effects of sinus arrhythmia on QT dispersion. Heart 80:77-79

27. Edge JA, Matthews DR, Dunger DB (1990) Failure of current insulin regimes to meet the overnight requirements of adolescents with insulin-dependent diabetes. Diabetes Res 15:109-112

28. Goodacre S, McLeod K (2002) ABC of clinical electrocardiography: Paediatric Electrocardiography. BMJ 324:1382-1385

29. Durakovic Z, Durakovic A, Korsic M (1994) Changes of the corrected QT interval in the electrocardiogram of patients with anorexia nervosa. Int J Cardiol 45:115-120

30. Choy AM, Lang CC, Chomsky DM, Rayos GH, Wilson JR, Roden DM (1996) Normalization of acquired QT prolongation in humans by intravenous potassium. Circulation 96:2149-2154

31. Marfella R, Rossi F, Giugliano D (2001) Hyperglycemia and QT interval: time for re-evaluation. Diabetes Nutr Metab 14:63-65
32. Harris ND, Heller SR (1999) Sudden death in young patients with Type 1 diabetes: a consequence of disease, treatment or both? Diabetic Med 16:623-624

33. Zanone MM, Favaro E, Catalfamo E (2003) Autonomic function and autoantibodies to autonomic nervous tissues at follow-up in a cohort of young patients with type 1 diabetes. Effects of serum from diabetic patients on human adrenergic cells. J Neuroimmunol 139:66-75

34. Karavanaki K, Baum JD (2003) Coexistence of impaired indices of autonomic neuropathy and diabetic nephropathy in a cohort of children with type 1 diabetes mellitus. J Pediatr Metab 16:79-90

35. Chessa M, Butera G, Lanza GA et al. (2002) Role of heart rate variability in the early diagnosis of diabetic autonomic neuropathy in children. Herz 27:785-790

36. Cahill M, Eustace P, de Jesus V (2001) Pupillary autonomic denervation with increasing duration of diabetes mellitus. Br J Ophthalmol 85:1225-1230

37. Karavanaki K, Davies AG, Morgan MH, Baum JD (1997) Autonomic function in a cohort of children with diabetes. J Pediatr Endocrinol Metab 10:599-607

38. Robinson RT, Harris ND, Ireland RH, Macdonald IA, Heller SR (2004) Changes in cardiac repolarization during clinical episodes of nocturnal hypoglycaemia in adults with type 1 diabetes. Diabetologia 47:312-315

39. Lee S, Harris N, Robinson RT, Yeoh L, Macdonald IA, Heller SR (2003) Effects of adrenaline and potassium on QTc interval and QT dispersion in man. Eur J Clin Invest 33:93-98

40. Sovik O, Thordarson H (1999) Dead-in-bed syndrome in young diabetic patients. Diabetes Care 22 [Suppl 2]:B40B42

41. Eckert B Agardh CD (1998) Hypoglycaemia leads to an increased QT interval in normal men. Clin Physiol 18:570575

42. Landstedt-Hallin L, Englund A, Adamson U, Lins PE (1999) Increased QT dispersion during hypoglycaemia in patients with type 2 diabetes mellitus. J Intern Med 246: 299-307

43. Robinson RT, Harris ND, Ireland RH, Lee S, Newman C, Heller SR (2003) Mechanisms of abnormal cardiac repolarization during insulin-induced hypoglycemia. Diabetes 52:1469-1474

44. Matyka KA, Crowne EC, Havel PJ, Macdonald IA, Matthews D, Dunger DB (1999) Counterregulation during spontaneous nocturnal hypoglycemia in prepubertal children with Type 1 diabetes. Diabetes Care 22:1144-115

45. Banarer S, Cryer PE (2003) Sleep-related hypoglycemiaassociated autonomic failure in type 1 diabetes: reduced awakening from sleep during hypoglycemia. Diabetes 52:1195-1203

46. Thordarson H, Sovik O (1995) Dead in bed syndrome in young diabetic patients in Norway. Diabet Med 12:782787 